\title{
MAGNETIC MICROSPHERE AN EMERGING DRUG DELIVERY SYSTEM
}

\author{
DIKSHA SHARMA*, ABHISHEK SHARMA
}

Department of Pharmacy, L. R. Institute of Pharmacy, Solan, Himachal Pradesh, India. Email: sharma25pharmacy@gmail.com

Received: 24 January 2017, Revised and Accepted: 06 March 2017

\begin{abstract}
The drug delivery system has been advanced to release the drug according to the body requirement during the entire period of treatment and also for the delivery at the targeted site. Several novel drug delivery systems have emerged encompassing different route of administration to achieve controlled and targeted drug delivery, magnetic microsphere carrier being one of them. Magnetic microsphere is an alternative to traditional radiation methods. As the traditional radiation methods use highly penetrating radiation that is absorbed throughout the body and cause side effects hence its use is limited. Therefore, a safe and effective alternate is needed like magnetic microsphere. The excessive circulating drug particles are minimized by this delivery system. Moreover, the aim of specific targeting is to enhance the effectiveness of drug delivery and at the same time to lessen the toxicity and side effects. Magnetic carriers receive magnetic responses to a magnetic field from incorporated materials that are used for magnetic microsphere are chitosan, dextran, etc. One of the most utilized magnetic microspheres is serum albumine whether from human or other suitable animals. Drug release from the albumin microsphere can be controlled by various stabilization procedures. Overall, the targeted magnetic microsphere is much valuable novel drug delivery system for what more work have to be done. By knowing the importance of all this, the present paper reviews the mechanism, preparation, and applications of magnetic microspheres. As the targeted drug delivery system implies selective and effective localization of drug into the target at therapeutic concentrations with limited access to non-target sites. Magnetic microspheres hold great promises for reaching the goal of controlled and site-specific drug delivery.
\end{abstract}

Keywords: Magnetic microspheres, Targeted drug delivery, Target sites, Body, Radiation, Efficiency, Toxicity, Reduce side effect.

(C) 2017 The Authors. Published by Innovare Academic Sciences Pvt Ltd. This is an open access article under the CC BY license (http://creativecommons. org/licenses/by/4. 0/) DOI: http://dx.doi.org/10.22159/ajpcr.2017.v10i6.17284

\section{INTRODUCTION}

Extensive labors have been dedicated in recent years to utilize potential pharmaceutical devices like novel drug delivery systems. Design and development of novel drug delivery system have two basics. First, it should distribute the drug in accordance with a predetermined rate, and second, it should release therapeutically effective quantity of drug at the site of action [1,2]. Conventional drug delivery undergoes from several drawbacks such as increased variation in the circulatory drug level, extra frequency of dosage administration, increased gastrointestinal irritation, and dose-related side effects. To solve these disadvantages, controlled release drug delivery systems have been planned for even and constant drug release over a prolonged period [3]. The steady drug release is achieved by use of different kinds of polymeric systems; biodegradable polymer microspheres are one of the most common types which are used as a targeted drug delivery systems. Microspheres can encapsulate many types of drugs with small molecules, proteins, and nucleic acids are easily administered through a syringe needle. They are normally biocompatible, can give high bioavailability, and are capable of constant release for long periods [4]. Magnetite offers a large potential for innovation in electronics, optoelectronics, magnetic storage, biomedical, Ferrofluid, separation, and magnetically guided drug carriers for targeting the therapy [5].

Magnetic microsphere is small particle, with diameter in the micrometer range $(1-1000 \mu \mathrm{m})$. Magnetic microsphere is occasionally referred to as microparticles. Magnetic polymer microspheres are generally composed of magnetic cores to make sure for strong magnetic response and polymeric shells to give positive functional groups and protect from particle aggregation [6]. Small amounts of drug targeted magnetically to restricted sites can replace large doses of drug that, using traditional administration methods, freely flow in the blood and beat the target site in a generalized way only. Moreover, drugs within the sphere are protected from breaking down during transport. Such drugs are targeted instead of distributed in blood and do not harm sensitive organs such as bone marrow. Magnetic microspheres are a substitute to traditional radiation methods which use extremely penetrating radiation that is absorbed throughout the body. Its use is limited by toxicity and side effects. Magnetic radioactive microspheres are applied in a way similar to non-radioactive spheres. A magnet, placed outside the body, is directed to the target site. The magnet can be a rod-shaped permanent magnet of any size or can be contained in equipment that looks like an open magnetic resonance imaging scanner. The loaded microspheres are introduced into a blood vessel and in as little as half an hour; they get collected at the target site to emit radiation which kills surrounding cancer cells. The remedial action is achieved usually in a couple of days or weeks depending on the material used. The treatment can be repeated if required. Spheres are prepared with microscopic magnetic particles, such as iron $[5,6]$, so these particles can be retained at the target site by the application of an external magnetic field of suitable strength. Magnetic fields are supposed to be safe to biological systems and adaptable to any part of the body.

\section{PRINCIPLES OF MAGNETIC DRUG TARGETING}

The principle of this treatment shows that the magnetic drug can be retained at or made to flow toward the target site by the application of an external magnetic field. Retention of magnetic carrier at target site will decrease reticuloendothelial clearance and increase site specificity [7].

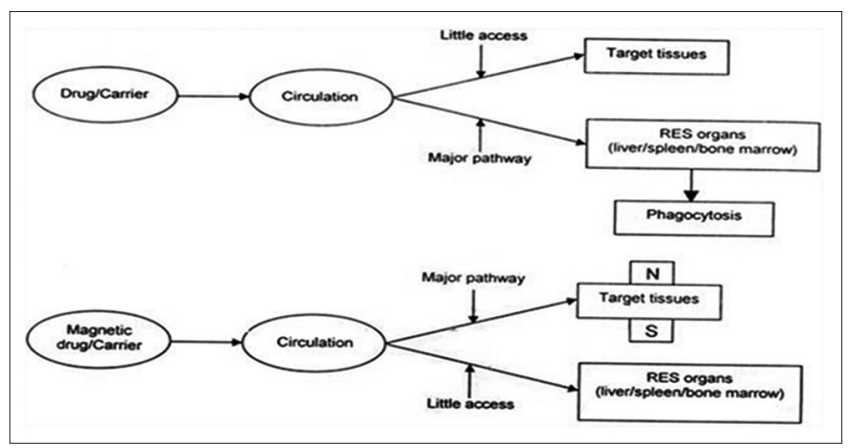




\section{HOW THE MAGNETIC MICROSPHERE WILL TARGET}

The major difficulty of cancer remedy is not the lack of better drug, but that such medicines are very difficult to concentrate in the tumor tissue without leading the toxic effect on normal nearby tissue or organs. Magnetic drug delivery by particular carriers is an efficient way of delivering a drug to a localized disease site. A drug or therapeutic radioisotope is encapsulated in a magneticakes place within area, to which the magnetic field is applied. The gathering of the carrier at the target site allows them to deliver the drug locally. Effectiveness of gathering of magnetic carrier on physiological carrier depends on physiological parameters, for example, particle size, surface characteristic, field strength, and blood flow rate. The magnetic field helps to flow the magnetic carrier into the targeted area. Large concentration of chemotherapeutic agents gets collected near the target site without any toxic effect to normal neighboring tissue or to whole body. It is possible to replace large amounts of drug targeted magnetically to localized disease site, reaching effective and up to several fold increased drug levels [8].

\section{Magnetite $\left(\mathrm{Fe}_{3} \mathrm{O}_{4}\right)$}

Magnetic particles consist of one or more magnetic cores with a coating matrix of polymers, silica or hydroxylapatite with terminal functionalized groups. The magnetic core generally consists of magnetite. Magnetite is magnetic oxide of iron and is a blend of the two oxides, $\mathrm{FeO}$ and $\mathrm{Fe}_{2} \mathrm{O}_{3}$. It is also referred to as ferrous ferrite. It gives magnetic property just like pure iron and exists in the form of fine particles and has been used for various application in vivo like in transmission radiography, as contrast gastrointestinal agent, for inducing clotting in arteriovenous malformation, as a tracer of blood flow and in radionuclide angiography. Iron particles with small diameter can pass through small capillaries in the body [7].

\section{Preparation of magnetite from a-FeOOH (goethite) or $\mathrm{FeO}$}

Here, the researchers flushed nitrogen gas through double-necked round-bottom flask $(500 \mathrm{ml})$ fitted with a condenser. Then, they charge the flask with $8.9 \mathrm{~g}(0.1 \mathrm{~mol})$ of $\mathrm{FeO}, 9.94 \mathrm{~g}(0.05 \mathrm{~mol})$ of $\mathrm{FeCl}_{2} .4 \mathrm{H}_{2} \mathrm{O}$ along with $250 \mathrm{ml}$ deionized water and then $50 \mathrm{ml}$ of $2 \mathrm{M} \mathrm{NaOH}$ was added. After refluxing reaction mixture for $12 \mathrm{hrs}$ and during the transformation of the $\mathrm{pH}$, its $\mathrm{pH}$ fell from 14 (orange) into 8-9 (black precipitates) particles were washed with distilled water, filtered and dried under vacuum at room temperature $[9,10]$.

\section{SELECTION OF DRUGS FOR THE PREPARATION OF MAGNETIC MICROSPHERES}

The drug to be selected should be less dangerous, inexpensive because waste rate for it is $99.9 \%$ [11].

Specific examples of drugs are as follows:

Adriamycin, amphotericin B, membrane active agents, prostaglandins, and immunosuppressive agents [12].

\section{METHODS OF PREPARATION OF MAGNETIC MICROSPHERES}

\section{Continuous solvent evaporation}

Polymer encapsulated microspheres are synthesized on the basis of a continuous solvent evaporation technique. A solution of polymer, drug, and magnetite should be added to the volatile organic solvent, which forms auxiliary solution on stirring. The resulting solution should be maintained at temperature of $22-30^{\circ} \mathrm{C}$. The suspension of magnetic microspheres is formed and should be separated by centrifugation. The product is freeze-dried and stored at $4^{\circ} \mathrm{C}$ [13].

\section{Phase separation emulsion polymerization}

The briefly aqueous solution of polymer, drug, and magnetite is added to the vegetable oil and emulsified using emulsifying agent. Stir this solution using magnetic stirrer at 1,500 rpm for 2 minutes. The resultant is stabilized by heating at $100-150^{\circ} \mathrm{C}$. Cross-linking agent is injected dropwise into the resultant emulsion by continuous stirring. The magnetic microspheres should form in the oil suspension and then separated from oil by washing procedures. The product is needed to freeze-dried and stored at $4^{\circ} \mathrm{C}[14]$

\section{Multiple emulsion method}

In this method, the researchers added water dispersible magnetite with a poly(acrylic acid)-poly(ethylene glycol) (PEG/PAA) coating added to the bovine serum albumin (BSA) containing inner water phase. $0.2 \mathrm{ml}$ of a $1 \mathrm{mg} / \mathrm{ml} \mathrm{BSA}$ solution added to a $4 \mathrm{ml}$ mixture of dichloromethane and EA at a ratio of 3-1 containing $200 \mathrm{mg}$ of poly(lactic-co-glycolic acid) (first $\mathrm{w} / \mathrm{o}$ emulsion has been prepared using a suitable homogenizer in an ice bath at 26,000 rpm for 2.5 minutes). Then, $15 \mathrm{ml}$ of a $1 \%$ poly(vinyl alcohol) (PVA) solution poured directly into the primary emulsion and re-emulsified using the similar homogenizer under the similar conditions for 2.5 minutes. Then, the $\mathrm{W} / \mathrm{o} / \mathrm{w}$ emulsion immediately poured into a beaker containing $85 \mathrm{ml}$ of $1 \%$ PVA solution and stirred in a hood under an overhead propeller for $2 \mathrm{hrs}$, allowing the solvent to evaporate. Solidified microspheres harvested by centrifugation at $2500 \mathrm{rpm}$ for 10 minutes and washed with distilled water 3 times [15].

\section{Preparation of blank $\mathbf{P}($ styrene-itaconic acid-divinylbenzene)} (St-IA-DVB) microsphere

The inverse phase suspension polymerization method has been employed to prepare microspheres. The reaction has performed in a $250 \mathrm{ml}$ three-neck flask fitted with a mechanical stirrer. The continuous phase comprised $100 \mathrm{ml}$ of castor oil and $10 \mathrm{ml}$ of span 80 . Then, a determined amount of IA, St, DVB, and BIS were dissolved completely in dimethyl sulfoxide, and the organic phase was added drop wisely into the flask, with $70^{\circ} \mathrm{C}$ heating using an oil bath. Then, the initiator of ammonium persulfate was added drop wisely with syringe. The reaction was continued for $8 \mathrm{hrs}$ with continuous stirring. The resulting microspheres were separated by centrifugation. The centrifuged microspheres were washed firstly with diethyl ether, followed by deionized water at least three times. After 3 times purification, the microspheres were lyophilized.

Preparation of magnetic nanoparticles coated $P$ (St-IA-DVB) microspheres (MNPSID microspheres)

For this, the researchers took $10 \mathrm{mg}$ of blank microspheres and dispersed in $50 \mathrm{ml}$ deionized water with continuous stirring. To this, they added $50 \mu \mathrm{l}$ of $\mathrm{Fe}_{3} \mathrm{O}_{4}$ nanoparticles drop wisely in $2 \mathrm{ml}$ deionized water with ultrasonic dispersion. After blending and dispersing completely in the water was done. The reaction kept up for $4 \mathrm{hrs}$, and the product was washed with excess deionized water 3 times and centrifuged. The obtained MNPSID microspheres were freeze dried and then kept in $4^{\circ} \mathrm{C}$ before use [16].

\section{Cross-linking method}

Here, the following reagent is used as follows:

Reagents used: Acetate buffer used as solvent for the chitosan polymer; cross-linker: Glutaraldehyde Used as medium: Sodium hydroxide solution synthesis of magnetic fluid: A $35 \%(\mathrm{w} / \mathrm{v})$ ferrous sulfate solution, $54 \%(\mathrm{w} / \mathrm{v})$ ferric chloride solution and $36 \%(\mathrm{w} / \mathrm{v})$ sodium hydroxide solution were prepared using distilled water.

Then, the ferric salt and ferrous salt were mixed, stirred, and heated. The alkaline solution was added when the temperature reached $55^{\circ} \mathrm{C}$. The mixture was stirred for 30 minutes, and then $5 \mathrm{~g}$ of PEG-10000 was added. The temperature was raised to $80^{\circ} \mathrm{C}$ and maintained for 30 minutes. The mixture was then neutralized while cooling, and the magnetic fluid was prepared. $1 \%(\mathrm{w} / \mathrm{w})$ chitosan was dissolved in acetate buffer at $\mathrm{pH}$ 4.5. The dissolved chitosan was added dropwise on the magnetic fluid. Formed chitosan magnetic microspheres were washed with deionized water and soaked in 1, 3, and 5 mol \% glutaraldehyde solution for $2 \mathrm{hrs}$ and then washed with deionized water [17]. 


\section{Swelling and penetration method}

For swelling of polymer microparticles, researchers mixed $0.25 \mathrm{~g}$ of PS (Micron-size polystyrene) particles with $35 \mathrm{~mL}$ of a $\mathrm{N}$-methyl- 2-pyrrolidone (NMP)/water solution in a specific $\mathrm{v} / \mathrm{v}$ NMP - to-water ratio. In later preparations of magnetic microspheres, they added sodium dodecyl sulfate (SDS) to the NMP/water solution. Whenever SDS was used, to each NMP/water solution $0.025 \mathrm{~g}$ of SDS was added. The NMP/water mixture with PS spheres was left soaking for $24 \mathrm{hrs}$ at room temperature with stirring. To the mixture of PS sphere and NMP/water solution, $2.5 \mathrm{~mL}$ of the super paramagnetic nanoparticle dispersion ( $24 \mathrm{mg} / \mathrm{mL}$ or other specified concentration) was added at $30^{\circ} \mathrm{C}$ while shaking (at $140 \mathrm{r}$ /minutes) for 1-5 days to allow the magnetic nanoparticles to penetrate into the interior of the PS particles. After this they separated, the polymer particles from the solution by centrifugation. Finally, particles were sequentially washed with methanol, deionized water, and dried under vacuum at room temperature for 1-2 days to yield the magnetic polymer microspheres [18].

\section{Sonochemical method}

In this method, the microspheres are having iron oxide-filled and coated globular BSA. Here, the magnetic microspheres were prepared from BSA and iron penta carbonyl or from BSA and iron acetate. Protein microspheres have a number of biomedical application, i.e., use as echo contrast agents for sonography. The microsphere was formed by two ways (i) either by heat denaturation at various temperatures or (ii) by cross linking with carbonyl compounds in the ether phase. Cross linking was completed as the microspheres are formed by chemically cross-linking cysteine residues of the protein with $\mathrm{HO}_{2}$ radical formed around a nonaqueous droplet. The chemical cross-linking is known to be responsible for the formation of the microspheres. This is due to the chemical ejects of the ultrasound radiation on an aqueous medium [19-22].

\section{Storage}

Several precautions have to be kept in mind during the formation microsphere. These are

1. Microsphere suspensions should not be frozen, as freezing is likely to cause irreversible aggregation.

2. Cold storage $\left(2-8^{\circ} \mathrm{C}\right)$ is recommended to inhibit microbial growth. "Standard" (non-protein coated) microsphere suspensions do not contain an antimicrobial agent.

3. All suspensions should be handled using aseptic technique.

4. Continuous rolling (e.g. 3-5 r/minutes on a cell culture roller) is recommended to keep microspheres in suspension, without generating foam (foam may cause particle loss through bead entrapment).

5. If continuous rolling is not possible, particles should be thoroughly resuspended before use. Higher speed rolling (30-60 r/minutes for 2-4 hrs) is effective for the resuspension of settled material.

6. Again, rolling speed is intended to effectively resuspend the beads without generation of foam [23].

\section{BIODISTRIBUTION AND TISSUE CONCENTRATION OF MICROSPHERES}

In physiological solution containing $0.1 \%(\mathrm{w} / \mathrm{v})$ Tween 80 or a viscosity enhancing agent, such as $50 \%(\mathrm{w} / \mathrm{v})$ dextran, aggregation in target vessel can be minimized by injecting microspheres. For spheres smaller than $3 \mu \mathrm{m}$, initial (5-30 minutes) biodistribution is a function of:

a. The dose relative to the capacity of target capillaries.

b. The degree to which the magnetic field overlaps microvessels supplied by the injection vessel.

c. The extent of venous shunting before microspheres reach the field.

d. The flow rates in target vessels.

The large doses of trace-labelled spheres $(6.6 \mathrm{mg} / \mathrm{kg}$, sufficient to produce effective levels of $\mathrm{f}$ - met-leu-phe peptide) were infused intravenously in to rates over a 3 minutes interval in a study. Here, the infusion was allowed to travel at native flow rates into the central venous system through the right heart in to bath lungs. The movement was captured by a $0.55 \mathrm{~T}$ magnetic field (gradient of $0.01 \mathrm{~T} / \mathrm{mm}$ ), which enveloped the right thorax and the middle half of the left thorax. In the later study, the splenic and renal concentrations of the spheres were reported to be slightly higher because of greater vascular shunting due to incomplete magnetic envelopment of the left lung.

\section{MAGNETICALLY MODULATED SYSTEMS AND DEVICES}

Magnetically modulated polymeric controlled drug delivery systems that deliver the drugs at increased rate on demand have been developed extensively in recent years. These systems consist of polymeric matrix, in which drug powder is discrete. The polymeric matrix consists of ethylene vinyl acetate copolymer (EVAc) with some magnetic beads. The beads used are either (i) magnetic steel beads composed of iron (79\%), chromium (17\%), carbon (1\%), manganese (1\%), silicon (1\%), molybdenum $(0.75 \%)$, and phosphorus $(0.04 \%)$ or (ii) small amount of samarium cobalt magnets. These systems are formulated by adding approximately $50 \%$ of drug-polymer mixture to a glass mold, which is cooled to $-80^{\circ} \mathrm{C}$ using dry ice, after this the magnetic particles, are added followed by the remaining drug-polymer mixture.

In case of in vivo experiment, the magnetic tablets are placed in glass vials. An oscillating external magnetic field, which is generated by a device that rotates the permanent magnets below the vials, controls the release rates.

\section{Mechanism}

The release of macromolecules from EVAc systems without magnetic beads suggests that molecules with molecular weight $>300$ cannot permeate the polymer. The direct merging of macromolecules in the polymer-macromolecule using cast method caused a tortuous and compound sequence of pores development in the matrix. The release rates are dogged by factors affecting infiltration of water into the polymer and drug out of these pores.

\section{Drug release}

Traditional methods failed to check drug, due to reduction in circulating free drug levels by a factor exceeding 100 when magnetic targeting is used (Ranney, 1985). Even the blood levels monitored by some possible methods do not give reasons when correlated with tissue levels. Estimates of free drug level can be made at dissimilar intervals from the concurrent interpretation of the results obtained from carrier localization studies in tissue and drug release profile in vitro. This method is simple and ignores some factors that influence drug release from carrier in vivo like enzymatic digestion of microspheres accelerated drug release and retro diffusion of the drug back to microvessel enhances drug clearance. The accurate and precise results can be obtained by measuring the drug levels of the target site for every using magnetic resonance methods [7]

\section{CHARACTERIZATION OF MAGNETIC MICROSPHERES}

1. Particle size and size distribution

The particle size of microsphere has been determined by using an optical microscope with the aid of a calibrated ocular micrometer. Here, total 100 particles were measured for size and then the average of these particles taken as average particle size.

2. Surface characterization

Surface characterization can be determined by using:

a. High-resolution microscopy

b. Scanning electron microscopy (SEM)

c. Scanning tunneling microscopy

3. Surface charge analysis

They can be achieved by using:

a. Micro electrophoresis

b. Laser Doppler anemometry.

4. Density

a. Tapped density

It is determined by pouring accurately weighed microspheres in measuring cylinder and tapped 100 times from a stable height 
to determine the tapped volume and finally tapped density is to be calculated.

True density = weight of powder/tapped volume of powder

b. Bulk density

It is determined by pouring accurately weighed microspheres in measuring cylinder and thus determining its bulk volume.

Bulk density = weight of powder/bulk volume of powder

5. Flow properties

Flow properties are measured by following ways:

a. Angle of repose

Angle of repose is the angle that a static heap of particles makes with the horizontal. The flow properties of microspheres can be determined by fixed funnel flow method, which is used to calculate angle of repose.

$$
\tan \theta=\mathrm{h} / \mathrm{r}
$$$$
\theta=\tan ^{-1} \mathrm{hr} / \mathrm{r}
$$

b. Hausner ratio

It is determined from the ratios of tapped density and bulk density. Hausner ratio $=$ tapped density/bulk dendity

6. Hardness

Hardness is defined as the force requires breaking the microsphere. It can be tested by using Monsanto hardness apparatus.

7. Friability

Hardness $=$ final reading - initial reading

For this, a rosche Friabilator is used for determining the friability.

$\%$ Friability $=$ initial weight - final weight $/$ initial weight $\times 100$

8. Surface area

9. Porosity

10. Drug content

11. Drug release profiles [24].

\section{Evaluation of magnetic microspheres}

\section{IR spectroscopic studies}

The IR spectra of the free drug and the microspheres were reported. The matching peaks equivalent to the functional groups and albumin (BSA, egg albumin, human serum albumin) features confirm that the polymer and the method of preparation has not affected the drug stability.

\section{Thin layer chromatographic (TLC) studies}

The TLC method has been followed for testing the drug stability in prepared microsphere. The Rf values of the prepared microspheres can be compared with the $\mathrm{Rf}$ value of the pure drug. The values point to the drug constancy.

\section{Surface topography by SEM}

SEM of the microspheres illustrates the surface morphology of the microspheres such as their shape and size.

\section{Particle size distribution of prepared microspheres}

The size of the ready microspheres can be measured by the optical microscopy way using calibrated stage micrometer for randomly selected samples of all the formulations.

\section{Drug entrapment capacity}

Effectiveness of drug entrapment for each batch can be calculated in terms of percentage drug entrapment as per the following formula:

$\%$ Entrapment $=($ actual content $/$ theoretical content $) \times 100$

\section{In vitro release studies}

In-vitro release studies can be achieved according to USP type I dissolution apparatus at appropriate $\mathrm{pH}$ conditions. The temperature should be upheld at $37 \pm 0.5^{\circ} \mathrm{C}$ and the rotation speed of $100 \mathrm{rpm}$. Then, $5 \mathrm{ml}$ of sample should be withdrawn at various time intervals and restocked with an equal volume of fresh dissolution media. The drug content in the sample can be analyzed spectrophotometrically at specific wavelength (nm) [25].

\section{In vivo experiments}

In vivo study here used, implants containing EVAc embedded magnets and bovine zinc insulin. The implants are placed in diabetic rats for 2 months and blood glucose levels were monitored. The blood glucose level decline due to diffusion of insulin from the polymer and when the diabetic rat were exposed to an oscillating magnetic field; the blood glucose levels were further lowered from 50 to $200 \mathrm{mg} / \mathrm{dl}$ below this basal level; this is since of magnetic field conditions. The result was confirmed by radioimmunoassay.

\section{Advantages of magnetic microspheres}

1. Therapeutic responses in target organs at only one-tenth of the free drug dose.

2. Controlled drug release in target tissues for intervals of 30 minutes $30 \mathrm{hrs}$, as desired.

3. To avoid acute drug toxicity directed against endothelium and normal parenchymal cells.

4. Adaptable to any part of the body.

Disadvantages of magnetic microspheres

1. It is an expensive, technical approach and need specialized manufacture and quality control system.

2. It needs specialized magnet for targeting, for monitoring, and trained personnel to perform procedures.

3. Magnets must contain relatively constant gradients, to avoid focal over- dosing with toxic drugs.

4. A large fraction (40-60\%) of the magnetite, which is entrapped in carriers, is deposited permanently in tissues.

\section{Applications of magnetic microsphere}

Magnetic microsphere carriers comprise large function in the field of bioengineering and biomedicine.

- Useful in the field of biological and biomedical developments.

- Drugs targeting, enzyme immobilization, cell isolation, and protein purification.

- Magnetic vehicles are better for delivery of therapeutic agents as they can be targeted to exact locations in the body through the application of a magnetic field.

- The magnetic localization of a therapeutic agent reducing or eliminating the systemic drug side effects.

- It is used in drug innovation, molecular targeting, DNA analysis, proteomics, and understanding the way of cell cycle regulation.

- As a chemotherapeutical agent to use in the treatment of cancer

- For stem cell extraction and bone marrow purging [26-27].

\section{Chemoattractant microspheres}

Neutrophil Chemoattractant was first selected biomodulator for magnetic targeting due to its property when administered freely in circulation at a concentration beyond $2 \times 10^{-7} \mathrm{M}$. It involves a sitespecific and local delivery to modulate inflammation and is a small, bacterially derived peptide, stable during microsphere preparation. It can be captured successfully in microparticles. The microsphere has been used either in disease modeling or clinical therapy.

In pulmonary medicine, these delivery systems are used as:

- An experimental method to test novel agents so as to prevent neutrophil degradation of lung elastine in smokers.

- A way of studying the involvement of acute alveolar damage to acute type respiratory distress syndrome.

- And as an adjuvant method in treating invasive pulmonary aspergillosis suffering patients.

\section{CONCLUSION}

Over the years, magnetic microsphere has been investigated for targeted drug delivery especially magnetic targeted chemotherapy due to their better tumor targeting. Targeted drug delivery is an effective method to 
assist the drug molecule to reach preferably to the desired site. It gives the idea that drugs reach the right site in the body, at the right time, at the right concentration. It does not exert side effects, neither on its way to the therapeutic target, not at the target site, nor during the clearance process. Thus, magnetic microspheres have the potential for these objectives. The main advantage of this technique is the reduction in the dose and side effects of the drug. The magnetic targeted chemotherapy has better tumor targeting, therapeutic efficacy, and lower toxicity. As the targeted drug delivery system implies selective and effective localization of drug into the target at therapeutic concentrations with limited access to non-target sites. Magnetic microspheres hold great promises for reaching the goal of controlled and site-specific drug delivery.

It is a demanding area for future research in the drug targeting so more researches, long-term toxicity study, and characterization determinations to give guarantee for the upgrading of magnetic drug delivery system. The future holds a lot of promises in magnetic microspheres, and by more study, this will be developed as novel and efficient approach for targeted drug delivery system.

\section{REFERENCES}

1. Peppas NA, Hilt JZ, Khademhosseini A, Langer R. Hydrogels in biology and medicine: From molecular principles to bio-nanotechnology. Adv Mater 2006; 18:1345-60.

2. Huang X, Brazel CS. On the importance and mechanisms of burst release in matrix-controlled drug delivery systems. J Control Release 2001;73(2-3):121-36.

3. Bhattarai N, Gunn J, Zhang M. Chitosan-based hydrogels for controlled, localized drug delivery. Adv Drug Deliv Rev 2010;62(1):83-99.

4. Kim KK, Pack DW. Microspheres for drug delivery. Biol Biomed Technol 2006;1:19-20.

5. Bergemann C, Muller-Schulte D, Oster J, Brassard L, Lubbe AS. Magnetic ion-exchange nano - And microparticles for medical, biochemical and molecular biological applications. J Magn Magn Mater 1999;194:45-52.

6. Asmatulu R, Zalich MA, Claus RO. Synthesis, characterization and targeting of biodegradable magnetic nanocomposite particles by external magnetic fields. J Magn Magn Mater 2005;292:108-19.

7. Vyas SP, Khar RK. Targeted and Controlled Drug Delivery. New Delhi: CBC Publisher \& Distributors; 2004. p. 459-63.

8. Johansen L, Nustad K, Ørstavik TB, Ugelstad J, Berge A, Ellingsen T. Excess antibody immunoassay for rat glan-dular kallikrein. Monosized polymer particles as the prefered solid phase material. J Immunol Methods 1983;59:255-64.

9. Lang C, Schuler D. Biogenic nanoparticles: Production, characterization, and application of bacterial magnetosomes. J Phys Condens Matter 2006;18:S2815-28.
10. Weckler B, Lutz HD. Magnetic microsphere an emerging drug delivery system. Solid State Inorg Chem Eur J Mol Struct 1998;35:531-44.

11. Gallo JM, Gupta PK, Hung CT, Prrier DG. Evaluation of drug delivery following the administration of magnetic albumin microspheres containin adriamycin to the rat. J Pharm Sci 1989;78:190-4.

12. Available from: http://www.bangslabs.com/technotes/201a. pdf accessed on 25/02/2011.

13. Lachman LA, Liberman HA, Kanig JL. The Theory and Practice of Industrial Pharmacy. Mumbai, India: Varghese Publishing House; 2002. p. 414-5.

14. Salim MD, Shukla VK, Bhardwaj V, Garg VK, Sharma PK. Magnetic microspheres as a magnetically targeted drug delivery system. J Glob Pharm Technol 2010;2(3):36-46

15. Collins AE, Deasy PB. Bioadhesive lozenge for the improved delivery of cetylpyridinium chloride. J Pharm Sci 1990;79(2):116-9.

16. Wang KE, Xing JF, Li XY, Fu Q, Li WF. Fabrication of novel magnetic nanoparticles-coated $\mathrm{P}$ (styrene-itaconic acid-divinylbenzene) microspheres. Carbohydr Polym 2009;321:1951-4.

17. Nasra MK, Mohamed MM, Elblbesy MA, Hefney BA. Preparation of biocompatible magnetic microspheres with Chitosan. J Biomater Nanobiotechnol 2011;2:194-200.

18. Chung TH, Lee WC. Preparation of styrene-based, magnetic polymer microspheres by a swelling and penetration process. React Funct Polym 2008;68:1441-7.

19. Keller MW, Feinstein SB. In: Kerber RE, editor. Echocardiography in Coronary Artery Disease. New York: Churchill Livingstone; 1988. p. $443-65$.

20. Suslick KS. The chemistry of ultrasound, encyclopaedia britannica yearbook of science and the future. Britannica. Chicago: Encyclopædia Britannica, Inc.; 1994. p. 138-55

21. Cax X, Prozorov R, Koltypin Y, Kataby G, Felner I, Gedanken A. Annealing study of $\mathrm{Fe} 2 \mathrm{O} 3$ nanoparticles: Magnetic size effects and phase transformations. J Mater Chem 1997;12:402-7.

22. Cax X, Koltypin Y, Prozorov R, Felner I, Gedanken A. Preparation and characterization of amorphous nanometer sized $\mathrm{Fe} 3 \mathrm{O} 4$ powder. J Mater Chem 1997; 7:1007-9.

23. Yeung YA, Wittrup KD. Quantitative screening of yeast surfacedisplayed polypeptide libraries by magnetic bead capture. Biotechnol Prog 2002;18(2):212-20.

24. Le B, Shinkai M, Kitade T, Honda H, Yoshida J, Wakabayashi T. Medical applications of functionalized magnetic nanoparticles. J Chem Eng Jpn 2001;34:66-72.

25. Chan LW, Heng PW. Effects of poly (vinylpyrrolidone) and ethylcellulose on alginate microspheres prepared by emulsification. J Microencapsul 1998;15(4):409-20.

26. Nachts S, Martin K. The Microsponges: A Novel Topical Programmable Delivery Formulation. New York: Marcel Dekker Inc.; 1990. p. 299.

27. Lea T, Vartdal F, Davies C, Ugelstad J. Magnetic monosized polymer particles for fast and specific fractionation of human mononuclear cells. Scand J Immunol 1985;22(2):207-16. 